\title{
CH AS A MOLECULAR GAS TRACER AND C-SHOCK TRACER ACROSS A MOLECULAR CLOUD BOUNDARY IN TAURUS
}

\author{
DUO XU (许铎) $1,2,4 *$, Di LI (李菂) $1,3 *$ \\ ${ }^{1}$ National Astronomical Observatories, Chinese Academy of Sciences, A20 Datun Road, Chaoyang District, Beijing 100012, China \\ ${ }^{2}$ University of Chinese Academy of Sciences, Beijing 100049, China \\ ${ }^{3}$ Key Laboratory for Radio Astronomy, Chinese Academy of Sciences, China \\ ${ }^{4}$ Current address: Department of Astronomy, University of Massachusetts, Amherst, MA 01003, USA
}

\begin{abstract}
We present new observations of all three ground-state transitions of the methylidyne $(\mathrm{CH})$ radical and all four ground-state transitions of the hydroxyl $(\mathrm{OH})$ radical toward a sharp boundary region of the Taurus molecular cloud. These data were analyzed in conjunction with existing CO and dust images. The derived $\mathrm{CH}$ abundance is consistent with previous observations of translucent clouds $\left(0.8 \leq A_{v} \leq 2.1 \mathrm{mag}\right)$. The $X(\mathrm{CH})$-factor is nearly a constant at $(1.0 \pm 0.06) \times 10^{22} \mathrm{~cm}^{-2} \mathrm{~K}^{-1} \mathrm{~km}^{-1} \mathrm{~s}$ in this extinction range, with less dispersion than that of the more widely used molecular tracers $\mathrm{CO}$ and $\mathrm{OH}$. $\mathrm{CH}$ turns out be a better tracer of total column density in such an intermediate extinction range than $\mathrm{CO}$ or $\mathrm{OH}$. Compared with previous observations, $\mathrm{CH}$ is overabundant below 1 mag extinction. Such an overabundance of $\mathrm{CH}$ is consistent with the presence of a C-shock. CH has two kinematic components, one of which shifts from 5.3 to $6 \mathrm{~km} \mathrm{~s}^{-1}$, while the other stays at $6.8 \mathrm{~km} \mathrm{~s}^{-1}$ when moving from outside toward inside of the cloud. These velocity behaviors exactly match with previous $\mathrm{OH}$ observation. The shifting of the two kinematic components indicates colliding streams or gas flow at the boundary region, which could be the cause of the C-shock.

Subject headings: ISM: evolution ISM: clouds - ISM: individual objects (Taurus) - molecules: ISM
\end{abstract}

\section{INTRODUCTION}

The $3.3 \mathrm{GHz} \Lambda$-doubling lines of the methylidyne radical $\mathrm{CH}$ have been commonly used to trace low density gas in diffuse clouds and at the boundaries of dense clouds (e.g. Magnani et al. 1989; Magnani \& Onello 1993). They have also been observed extensively toward translucent clouds, bright limbed clouds, outflows, and dark clouds (e.g. Lang \& Wilson 1978; Sandell et al. 1980, 1981; Mattila 1986; Jacq et al. 1987; Sandell et al. 1988; Magnani et al. 1992). Many of such surveys have shown a linear correlation between the $\mathrm{CH}$ column density and the visual extinction in diffuse and translucent clouds (e.g. Hjalmarson et al. 1977; Mattila 1986; Magnani \& Onello 1993; Magnani et al. 2005). CH is therefore recognized as a powerful tracer of molecular hydrogen, tightly correlated with molecular hydrogen in terms of column density $[\mathrm{CH}] /\left[\mathrm{H}_{2}\right]=3.5 \times 10^{-8}$ (Sheffer et al. 2008). However, in a high spatial resolution observation of two high-latitude translucent clouds MBM 3 and 40 with the Arecibo Telescope, Chastain et al. (2010) found a slight spatial offset between the distribution of $\mathrm{CH}$ and that of $\mathrm{CO}$, possibly due to the chemical evolution of carbon. At higher visual extinctions, $\mathrm{CH}$ is consumed by the carbon chemistry in relatively dense molecular environments (Mattila 1986; Viala 1986). Thus, CH observations cannot supplant CO for studying high-visual-extinction clouds such as dark clouds or giant molecular clouds.

Owing to different formation routes, $\mathrm{CH}$ can exist in both high and low density gas. In high density gas, $\mathrm{CH}$ can be formed through the following chains of reactions

*Email: dxu@astro.umass.edu, dili@nao.cas.cn
(Black \& Dalgarno 1973):

$$
\begin{array}{r}
\mathrm{C}^{+}+\mathrm{H}_{2} \rightarrow \mathrm{CH}_{2}^{+}+h \nu \\
\mathrm{CH}_{2}^{+}+\mathrm{H}_{2} \rightarrow \mathrm{CH}_{3}^{+}+\mathrm{H} \\
\mathrm{CH}_{2}^{+}+e^{-} \rightarrow \mathrm{CH}+\mathrm{H} \\
\mathrm{CH}_{3}^{+}+e^{-} \rightarrow \mathrm{CH}+\mathrm{H}_{2} .
\end{array}
$$

When the amount of ionized carbon ([C II]) is substantial, $\mathrm{CH}$ formation is believed to be triggered by the radiative synthesis of $[\mathrm{C} \mathrm{II}]$ with vibrationally excited molecular hydrogen $\mathrm{H}_{2}$ (as described in Equation (1)) in the outer layers of photodissociation regions (PDRs), where the chemical evolution is dominated by UV radiation. Observationally, the evidence for the above equations has been inconclusive (see e.g. the $\mathrm{CH}_{3}^{+}$observation toward Cyg OB2 by Indriolo et al. 2010). In lower density material $\left(n_{H} \sim 50 \mathrm{~cm}^{-3}\right)$, $\mathrm{CH}$ can also be produced through $\mathrm{CH}^{+}$synthesis,

$$
\begin{aligned}
\mathrm{C}^{+}+\mathrm{H} & \rightarrow \mathrm{CH}^{+}+h \nu \\
\mathrm{C}^{+}+\mathrm{H}_{2}+0.396 \mathrm{eV} & \rightarrow \mathrm{CH}^{+}+\mathrm{H} \\
\mathrm{CH}^{+}+e^{-} & \rightarrow \mathrm{CH}+h \nu,
\end{aligned}
$$

propelled by MHD shocks (Draine \& Katz 1986; Pineau des Forets et al. 1986). CH is thus considered to be a tracer of MHD shocks, especially the C-type shocks (Flower \& Pineau des Forets 1998). Draine \& Katz (1986) made theoretical calculations on the abundance of $\mathrm{CH}$ when a C-type shock propagates into a diffuse cloud with $n_{\mathrm{H}}=50 \mathrm{~cm}^{-3}$, which may help us identify the presence of C-shocks. However, Gredel et al. (1993) and Crawford (1995) observed optical $\mathrm{CH}$ and $\mathrm{CH}^{+}$lines in several stars but found their line profiles to be inconsistent with shock theories. They believed that turbulent chemistry gives rise to the overabundance of $\mathrm{CH}$ and 
$\mathrm{CH}^{+}$in these clouds. Morris et al. (2016) found UV irradiation rather than shock chemistry playing a key role in $\mathrm{CH}^{+}$formation in Orion BN/KL.

The boundary of molecular clouds is the region in which C-shock may take place (Xu et al. 2016). A clear example of cloud boundaries can be found in Taurus (Goldsmith et al. 2008), northeast of the TMC1 region with a visual extinction ranging from 0.4 to $2.7 \mathrm{mag}$, and is thus an ideal target to study $\mathrm{CH}$ as a molecular tracer in the transition zone between diffuse and dense gas. $\mathrm{Xu}$ et al. (2016) have found evidence of the existence of C-shock across the Taurus boundary, such as the overabundance of $\mathrm{OH}$ at visual extinctions at or below $1 \mathrm{mag}$, the conjugate emission of $\mathrm{OH} 1612$ and 1720 $\mathrm{MHz}$ components, and the colliding streams or gas flow at the boundary region. All the evidence of C-shock across the Taurus boundary in Xu et al. (2016) is derived from $\mathrm{OH}$ spectra. Further spectral analysis of $\mathrm{CH}$ can provide insights into the presence of C-shock across the Taurus boundary.

We have carried out observations of the Taurus boundary in three $\mathrm{CH}$ transitions $(3335,3264$, and $3349 \mathrm{MHz}$, as shown in Figure 1) using the $305 \mathrm{~m}$ Arecibo Telescope. We made a total of five cuts 1.5 arcminutes apart across the boundary region each with 17 pointings (Figure 2). We describe the observations of $\mathrm{CH}$ and $\mathrm{OH}$ across the boundary region and the ${ }^{12} \mathrm{CO} J=1-0$, and ${ }^{13} \mathrm{CO} J=1-0$ map of the Taurus molecular cloud in Section 2. We analyze the $\mathrm{CH}$ spectrum and derive CH column density across the boundary in Section 3. We discuss the advantage of $\mathrm{CH}$ as a molecular tracer in Section 4. We compare the $\mathrm{CH}$ abundance with the C-shock model in Section 5. In Section 6 we summarize our results and conclusions from this study.

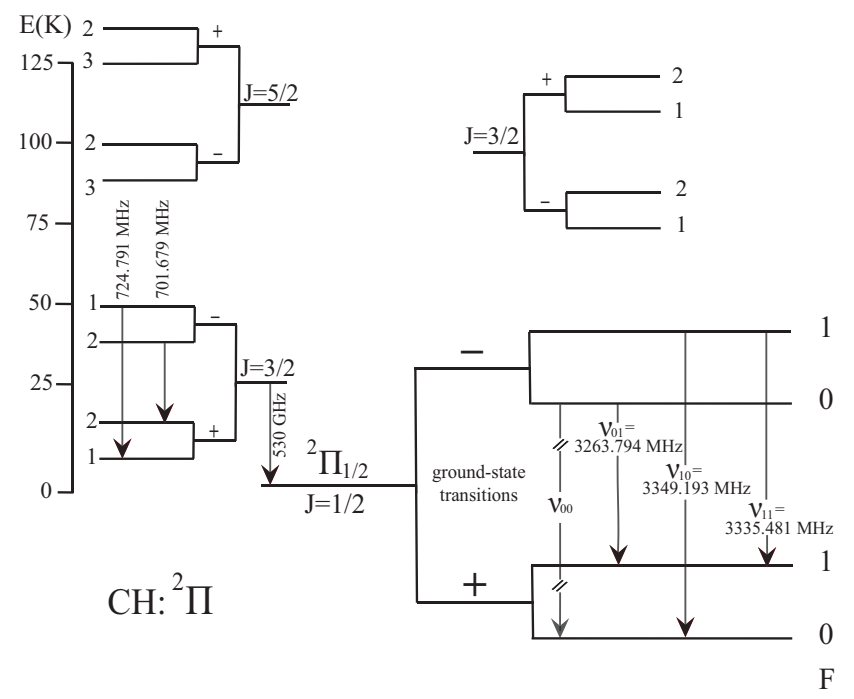

Figure 1. The energy levels of $\mathrm{CH}$ (not shown to scale). The intensity ratio between the allowed ground state transitions is $I\left(\nu_{11}\right): I\left(\nu_{10}\right): I\left(\nu_{01}\right)=2: 1: 1$ under LTE assumption.

\section{OBSERVATIONS AND DATA}

We carried out observations of the $\Lambda$-doubling lines of $\mathrm{CH}$ in the ${ }^{2} \Pi_{1 / 2}, J=1 / 2$ and the $\Lambda$-doubling lines of $\mathrm{OH}$ in the ${ }^{2} \Pi_{3 / 2}, J=3 / 2$ with the Arecibo Telescope
(Project a2813). We extracted ${ }^{12} \mathrm{CO} J=1-0$ and ${ }^{13} \mathrm{CO}$ $J=1-0$ data from the Five College Radio Astronomy Observatory (FCRAO) Taurus survey (Narayanan et al. 2008).

\subsection{CH Observations}

The $\mathrm{CH}$ observations were taken using the S-high receiver (3.0-4.0 GHz) on 2015 October 25-26 and November 24-26. We observed three $\Lambda$-doubling lines of $\mathrm{CH}$ in the ${ }^{2} \Pi_{1 / 2}, J=1 / 2$ (as shown in Figure 1 ) at the rest frequencies of 3335.481 (main line $F=1-1$ ), 3263.794 (lower satellite line $F=0-1$ ), and $3349.193 \mathrm{MHz}$ (upper satellite line $F=1-0$ ) with the total power ON mode. Spectra were obtained with the Arecibo WAPP correlator with three-level sampling and 8192 spectral channels for each line in each polarization. The spectral bandwidth was $3.13 \mathrm{MHz}$ for a channel spacing of about 381 $\mathrm{Hz}$, or $0.034 \mathrm{~km} \mathrm{~s}^{-1}$. The average system temperature was about $30 \mathrm{~K}$. The main beam of the antenna pattern had a full width at half maximum (FWHM) beam-width of $1.5^{\prime}$. Spectra were taken at $17 \times 5$ positions across the Taurus boundary region (TBR), as seen in Figure 2. An integration time of $450 \mathrm{~s}$ per position was used resulting in RMS noise level of about $0.038 \mathrm{~K}$. To get a higher signal-to-noise ratio, we smoothed the $\mathrm{CH}$ spectra to a velocity resolution of $0.14 \mathrm{~km} \mathrm{~s}^{-1}$, which yields a RMS noise level of about $0.019 \mathrm{~K}$.

\subsection{OH Observations}

The $\mathrm{OH}$ observations were taken using the L-band wide receiver $(1.55-1.82 \mathrm{GHz})$ on 2013 October $28-31$. We observed four $\Lambda$-doubling lines of $\mathrm{OH}$ in the ${ }^{2} \Pi_{3 / 2}, J=3 / 2$ at the rest frequencies of $1612.231,1665.402,1667.359$, and $1720.530 \mathrm{MHz}$ with the total power ON mode. Spectra were obtained with the Arecibo WAPP correlator with nine-level sampling and 4096 spectral channels for each line in each polarization. The spectral bandwidth was $3.13 \mathrm{MHz}$ for a channel spacing of about $763 \mathrm{~Hz}$, or $0.142 \mathrm{~km} \mathrm{~s}^{-1}$. The average system temperature was about $31 \mathrm{~K}$. The main beam of the antenna pattern had a FWHM beam-width of $3^{\prime}$. Spectra were taken at the same positions as those of $\mathrm{CH}$ across the $\mathrm{TBR}$, as seen in Figure 2. An integration time of $300 \mathrm{~s}$ per position was used resulting in a RMS noise level of about $0.027 \mathrm{~K}$.

\section{3. ${ }^{12} \mathrm{CO}$ and ${ }^{13} \mathrm{CO}$ Data}

The ${ }^{12} \mathrm{CO} \mathrm{J}=1-0$ and ${ }^{13} \mathrm{CO} \mathrm{J}=1-0$ observations were taken simultaneously between 2003 and 2005 using the $13.7 \mathrm{~m}$ FCRAO Telescope (Narayanan et al. 2008). The map is centered at $\alpha(2000.0)=04^{h} 32^{m} 44.6^{s}$, $\delta(2000.0)=24^{\circ} 25^{\prime} 13.08^{\prime \prime}$, with an area of $\sim 98 \mathrm{deg}^{2}$. The main beam of the antenna pattern has a FWHM beam-width of $45^{\prime \prime}$ for ${ }^{12} \mathrm{CO}$ and $47^{\prime \prime}$ for ${ }^{13} \mathrm{CO}$. The angular spacing (pixel size) of the resampled on-the-fly data is $20^{\prime \prime}$ (Goldsmith et al. 2008), which corresponds to a physical scale of $\approx 0.014 \mathrm{pc}$ at a distance of $D=140 \mathrm{pc}$. The data have a mean RMS antenna temperature of 0.28 $\mathrm{K}$ for ${ }^{12} \mathrm{CO}$ and $0.125 \mathrm{~K}$ for ${ }^{13} \mathrm{CO}$. There are 80 and 76 channels with 0.26 and $0.27 \mathrm{~km} \mathrm{~s}^{-1}$ spacing for ${ }^{12} \mathrm{CO}$ and ${ }^{13} \mathrm{CO}$, respectively.

\section{ANALYSIS}




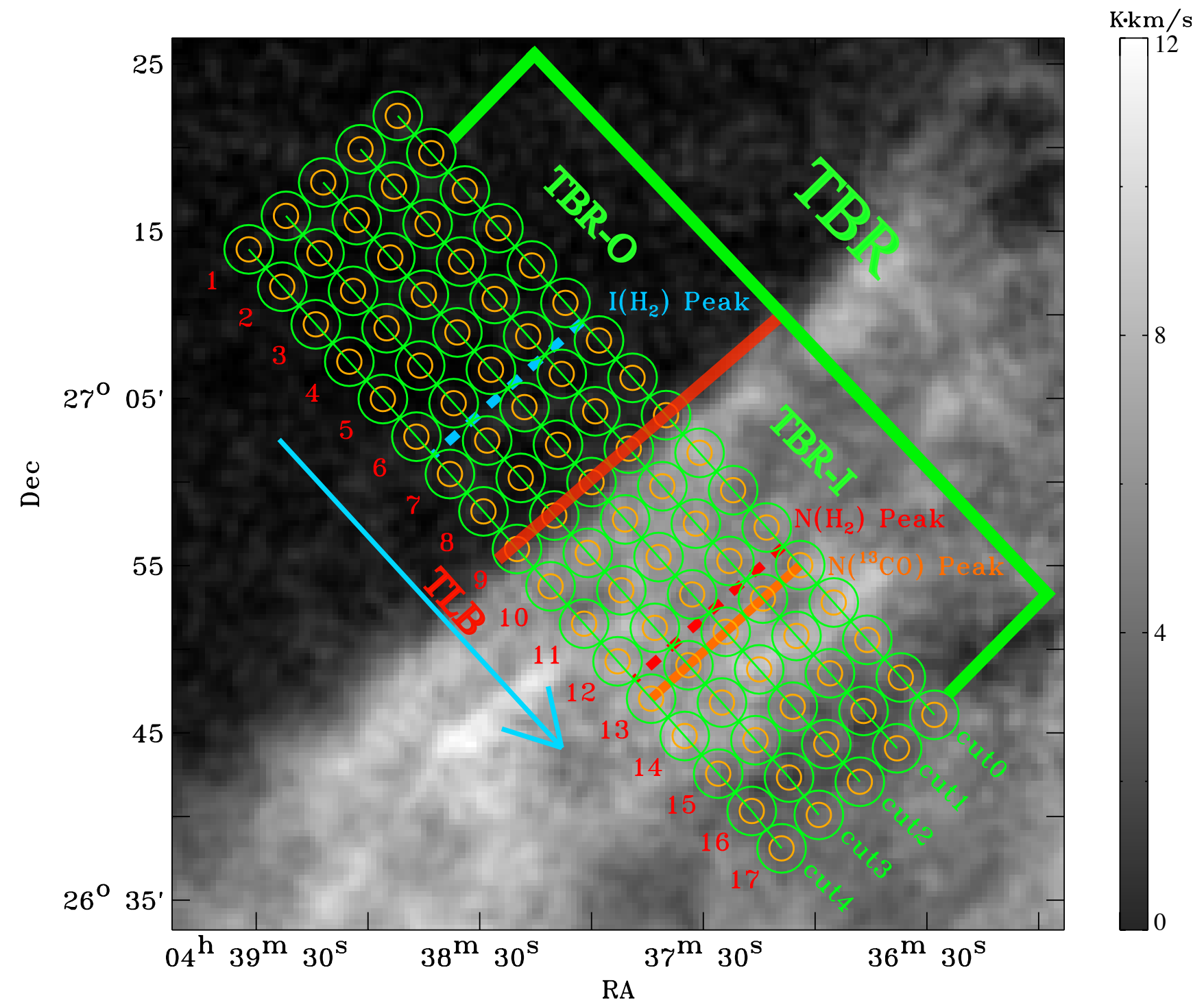

Figure 2. Boundary region in ${ }^{13} \mathrm{CO} \mathrm{J}=1-0$ peak intensity, with observed positions indicated. The $1.5^{\prime}$ yellow circle and the $3^{\prime}$ green circle indicate the telescope pointings for $\mathrm{CH}$ and $\mathrm{OH}$ observation, respectively. The numbers of positions are shown in the figure. The whole Taurus boundary region is denoted as TBR. The Taurus linear boundary (TLB) located at position 9 is shown as a red line. The outside and inside regions of the TBR are abbreviated as TBR-O and TBR-I, respectively. The peak intensity of the two lowest rotational transitions of $\mathrm{H}_{2}, \mathrm{~S}(0)$ and $\mathrm{S}(1)$, is located between position 6 and position 7 (Goldsmith et al. 2010). The peak column density of $\mathrm{H}_{2}$ is located between position 12 and position 13. The peak column density of ${ }^{13} \mathrm{CO}$ is located at position 13 . The arrow in the figure indicates the direction we present spectral line maps.

\subsection{Spectral Analysis}

The locations of the positions for the telescope pointing used to study the TBR are shown in Figure 2. To examine the transition zone with a higher signal-to-noise ratio, we averaged all five cuts of spectra of $\mathrm{CH} 3335$, 3264, $3349 \mathrm{MHz}, \mathrm{OH} 1612,1665,1667,1720 \mathrm{MHz},{ }^{12} \mathrm{CO}$ $\mathrm{J}=1-0$, and ${ }^{13} \mathrm{CO} \mathrm{J}=1-0$, as shown in Figure 3. The ${ }^{12} \mathrm{CO} \mathrm{J}=1-0$ and ${ }^{13} \mathrm{CO} \mathrm{J}=1-0$ spectra were convolved to the $\mathrm{OH}$ beam size of $3^{\prime}$ at each position. The emission lines of $\mathrm{CH}, \mathrm{OH},{ }^{12} \mathrm{CO} \mathrm{J}=1-0$, and ${ }^{13} \mathrm{CO} \mathrm{J}=1-0$ are well matched in velocity. In particular, the emission lines of $\mathrm{CH} 3335 \mathrm{MHz}$ and $\mathrm{OH} 1665 \mathrm{MHz}$ at positions 10-12 all have two components and are well matched in velocity as shown in Figure 4.

We fit a two-component Gaussian to the profiles of the
CH 3335, 3264, 3349, OH 1612, 1665, 1667, $1720 \mathrm{MHz}$ spectra and a single Gaussian to ${ }^{12} \mathrm{CO}$ and ${ }^{13} \mathrm{CO}$ spectra. The best fitting parameters of $\mathrm{CH} 3335 \mathrm{MHz}, \mathrm{OH}$ $1665 \mathrm{MHz}$ and $\mathrm{CO}$ are listed in Table 1-3, respectively. We show the spectra and the fitted profiles in Figure 3. $\mathrm{Xu}$ et al. (2016) have discussed the two components of OH $1665 \mathrm{MHz}$ across the TBR, which indicate the colliding streams or gas flows at the TBR. The CH $3335 \mathrm{MHz}$ spectra also have two components and are well matched with $\mathrm{OH} 1665 \mathrm{MHz}$ and $\mathrm{CO}$ in velocity across the TBR, as shown in Figure 5, which further confirm the assumption of colliding streams or gas flows across the TBR.

The shift in the single-component $\mathrm{CO}$ velocity is well correlated with the behavior of the $\mathrm{CH}$ velocity components. When the red $\left(6.5 \mathrm{~km} \mathrm{~s}^{-1}\right) \mathrm{CH}$ component 
is stronger in TBR-O, ${ }^{13} \mathrm{CO}$ peaks at $6.3 \mathrm{~km} \mathrm{~s}^{-1}$. In contrast, when the blue $\left(5.4 \mathrm{~km} \mathrm{~s}^{-1}\right) \mathrm{CH}$ component is stronger in TBR-O, ${ }^{13} \mathrm{CO}$ peaks at $5.7 \mathrm{~km} \mathrm{~s}^{-1}$. In Figure 4 and 5 , the red components of $\mathrm{CH}$ and $\mathrm{OH}$ gradually become fainter, and disappear at position 13. At the same time, the central velocities of blue components gradually shift from $5.4 \mathrm{~km} \mathrm{~s}^{-1}$ at position 9 to $5.8 \mathrm{~km}$ $\mathrm{s}^{-1}$ at position 13 , which indicates that the collision of two streams results in the final central velocity being located between the velocities of the two components. The central velocity of the final combined stream is located closer to the blue components, which have stronger emission lines in TBR-I. This is consistent with the assumption of different amounts of ${ }^{13} \mathrm{CO}$ emission at different velocities.

The change of the line width of $\mathrm{CH} 3335 \mathrm{MHz}, \mathrm{OH}$ $1665 \mathrm{MHz},{ }^{12} \mathrm{CO} \mathrm{J}=1-0$ and ${ }^{13} \mathrm{CO} \mathrm{J}=1-0$ along the cut direction is shown in Figure 6. The line width of the $\mathrm{CH}$ $3335 \mathrm{MHz}$ red component in TBR-O is almost a constant $\sim 1.8 \mathrm{~km} \mathrm{~s}^{-1}$. After merging with the blue component, the line width slightly decreases to $\sim 1.4 \mathrm{~km} \mathrm{~s}^{-1}$. The line width of $\mathrm{OH} 1665 \mathrm{MHz}$ remains almost constant across the TBR. The line width of ${ }^{12} \mathrm{CO}$, which continues to increase in TBR-O, behaves differently from that of $\mathrm{CH}$ $3335 \mathrm{MHz}$, which is almost constant in TBR-O.

Draine \& Katz (1986) pointed out that, owing to the different response of neutral and ionized species to the presence of a magnetic field, the $\mathrm{CH}^{+}$and $\mathrm{CH}$ formed in the hot post-shock gas can differ significantly in velocities. If shock waves propagate across the TBR, different neutral molecules obtain different velocities owing to their different cross section (e.g. Lambert \& Danks 1986; Gredel et al. 1993). We find a slightly different central velocities of $\mathrm{CH}, \mathrm{OH}$ and ${ }^{12} \mathrm{CO}$ across the TBR in Figure 5, which may be the result of the propagation of C-shock. More discussion of the probable C-shock across the TBR can be found in Section 5 .

\subsection{CH Column Density}

The TBR has a relatively low UV field between $\chi=0.3$ and 0.8 in units of the Draine's field (Flagey et al. 2009; Pineda et al. 2010). The gas kinetic temperature does not exceed $40 \mathrm{~K}$ even in the outermost layer of the TBR, and can be as low as $10 \mathrm{~K}$ in the inner part of the TBR. This means that almost all the $\mathrm{CH}$ molecules are populated in the ${ }^{2} \Pi_{1 / 2}, J=1 / 2 \Lambda$-doubling levels (as shown in Figure 1), and the populations of the higher $J$ levels are negligible. Although the gas kinetic temperature is approximately $40 \mathrm{~K}$ in the outermost layer of the TBR due to the heating by interstellar UV radiation, the density there is too low $\left(\sim 60 \mathrm{~cm}^{-3}\right)$ to excite $\mathrm{CH}$ even to the first rotationally excited state $(J=3 / 2)$. The critical density required to excite the lowest rotational transition $\left(J=3 / 2-1 / 2,530 \mathrm{GHz}\right.$ ) is as high as $10^{6} \mathrm{~cm}^{-3}$ (Sakai et al. 2012). Hence, practically all $\mathrm{CH}$ molecules are in the $J=1 / 2$ levels. For this reason, we only considered the $J=1 / 2$ levels for calculations of the partition function in the calculation of $\mathrm{CH}$ column density.

In most of the observation, the populations of the ${ }^{2} \Pi_{1 / 2}, J=1 / 2 \Lambda$-doubling levels can be inverted over a wide range of physical conditions causing weak masers producing negative excitation temperatures. In the case of the main line, the excitation temperature $T_{\mathrm{ex}, 11}$ for
$\mathrm{CH}$ is in the range $\sim-60$ to $-10 \mathrm{~K}$ (Hjalmarson et al. 1977; Genzel et al. 1979; Bujarrabal et al. 1984; Sandell et al. 1988; Liszt \& Lucas 2002).

We calculated the column density of $\mathrm{CH}$ assuming it to be optically thin, which is reasonable owing to the very small Einstein A-coefficients of the transitions $A=$ $1.94 \times 10^{-10} \mathrm{~s}^{-1}$ :

$$
N(\mathrm{CH})=2.82 \times 10^{14} \frac{1}{1-T_{\mathrm{bg}} / T_{\mathrm{ex}, 11}} \int T_{\mathrm{MB}}(3335) \mathrm{d} v,
$$

where $T_{\mathrm{MB}}(3335)$ is the main-beam brightness temperature of the main $\Lambda$-doubling line $(F=1-1)$ in ${ }^{2} \Pi_{1 / 2}$, $T_{\mathrm{bg}}$ is the cosmic background temperature, and $T_{\mathrm{ex}, 11}$ is the excitation temperature of the $F=1-1$ transition.

We have to choose a value for $T_{\mathrm{ex}, 11}$ to calculate the $\mathrm{CH}$ column density. As mentioned above, in most of the observation, $\mathrm{CH}$ emission can be weak masers yielding to negative excitation temperatures $T_{\mathrm{ex}, 11}$ in the range $\sim-60$ to $-10 \mathrm{~K}$. We adopted $T_{\mathrm{ex}, 11}=-15 \mathrm{~K}$ which is in the range of previously observed temperatures and also has also been widely used in diffused gas (Lang \& Wilson 1978; Liszt \& Lucas 2002). When $T_{\mathrm{ex}, 11}=-15 \mathrm{~K}$, the correction factor for excitation temperature,

$$
f_{\mathrm{ex}, \mathrm{bg}}=\frac{1}{1-T_{\mathrm{bg}} / T_{\mathrm{ex}, 11}},
$$

is about 0.85 . We parametrize the trend of $\mathrm{CH}$ column density across the TBR in a Gaussian profile, as shown in Figure 7. The trend of $\mathrm{CH}$ column density can be well described as

$N(\mathrm{CH})=2.6 \times 10^{13} \exp \left[-\left(\frac{A_{v}-2.0}{1.0}\right)^{2}\right]+1.6 \times 10^{13} \mathrm{~cm}^{-2}$.

When extinction exceeds 2 mag in TBR, CH column density starts to drop, indicating the consumption of $\mathrm{CH}$ via the carbon chemistry in relatively dense molecular environments, with more carbon locked into CO (Viala 1986).

When using the value of $T_{\mathrm{ex}, 11}=-60 \mathrm{~K}$ from Genzel et al. (1979), which is widely used for dark clouds, $f_{\text {ex }, \mathrm{bg}}$ increases by about $11 \%$. The uncertainty in $\mathrm{CH}$ column density associated with the assumptions of $T_{\mathrm{ex}, 11}$ is thus small.

The assumption that the $\Lambda$-doubling lines of $\mathrm{CH}$ are optically thin can be problematic, which can be seen from the relative intensities of the three components (Figure 3). Both satellite lines should be two times weaker than the main component, but the observed intensities of satellite lines are often larger. Furthermore, the intensity of the two observed satellite lines are not equal. Thus, we cannot precisely calculate the optical depth of $\mathrm{CH}$. The correction factor for optical depth is defined as

$$
f_{\tau}=\frac{\tau}{1-e^{-\tau}}
$$

where $\tau$ is the optical depth of the main $\Lambda$-doubling line. The line ratio between the satellite line and the main line ranges from 0.5 to 0.9 , resulting in $1 \leq f_{\tau} \leq 2.6$. Considering all the factors above (including $T_{\mathrm{ex}, 11}$ and $\tau)$, the $\mathrm{CH}$ column density can be underestimated by as much as a factor of 3 . 


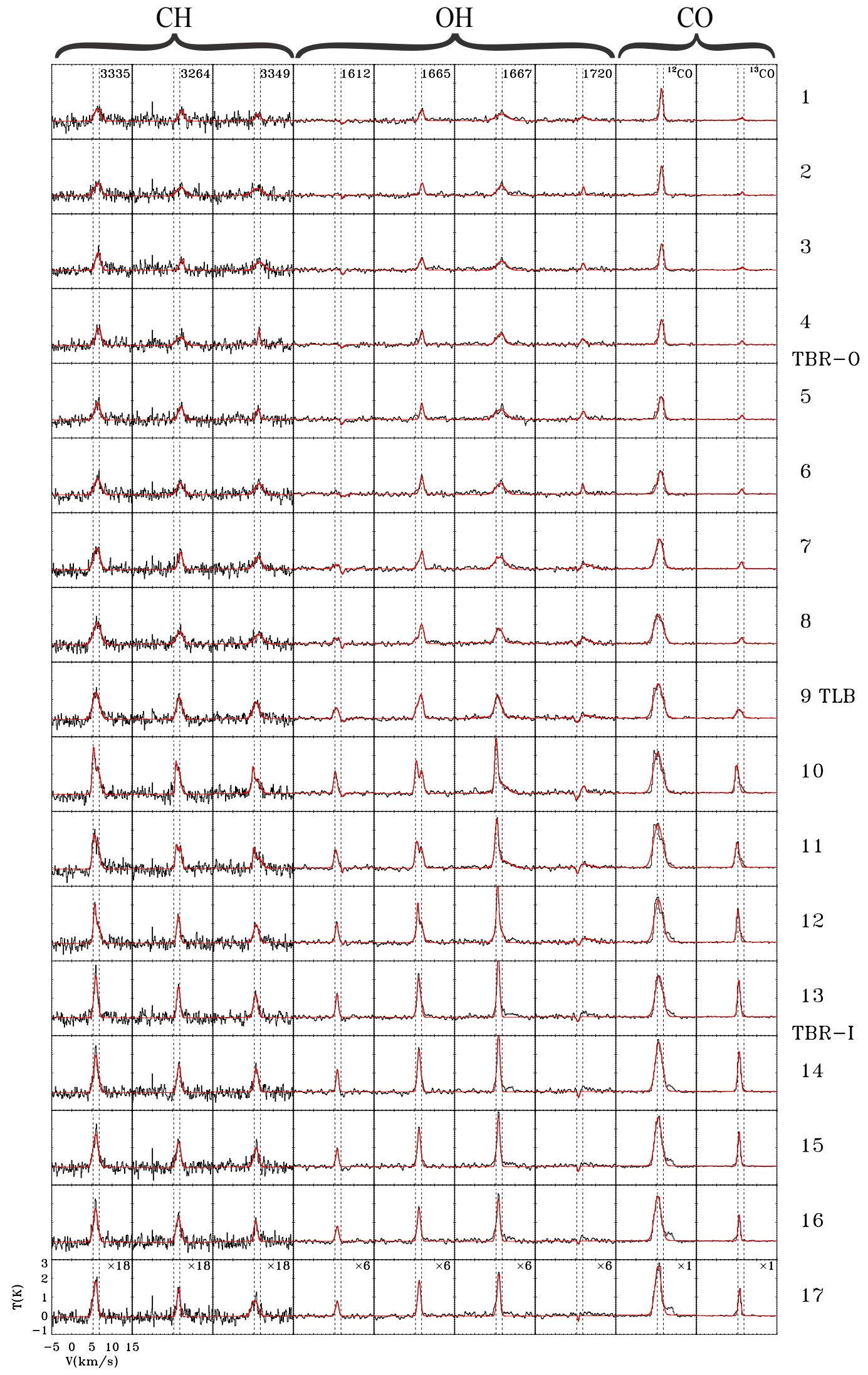

Figure 3. Average spectra of all five cuts of $\mathrm{CH} 3335,3264,3349 \mathrm{MHz}, \mathrm{OH} 1612,1665,1667,1720 \mathrm{MHz},{ }^{12} \mathrm{CO} \mathrm{J}=1-0$, and ${ }^{13} \mathrm{CO} \mathrm{J}=$ 1-0 overlaid with corresponding fitted Gaussian profiles (red curve). The ${ }^{12} \mathrm{CO} \mathrm{J}=1-0$ and ${ }^{13} \mathrm{CO} \mathrm{J}=1-0$ spectra were convolved to the $\mathrm{OH}$ beam size of $3^{\prime}$ at each position. We fitted the CH 3335, 3264, 3349 MHz, OH 1612, 1665, 1667, 1720 MHz spectra with two Gaussian components, and fitted the ${ }^{12} \mathrm{CO} \mathrm{J}=1-0$ and ${ }^{13} \mathrm{CO} \mathrm{J}=1-0$ spectra with single Gaussian component. The vertical dashed lines indicate the central velocities of the two components of $\mathrm{OH} 1665 \mathrm{MHz}$ at position 10. 


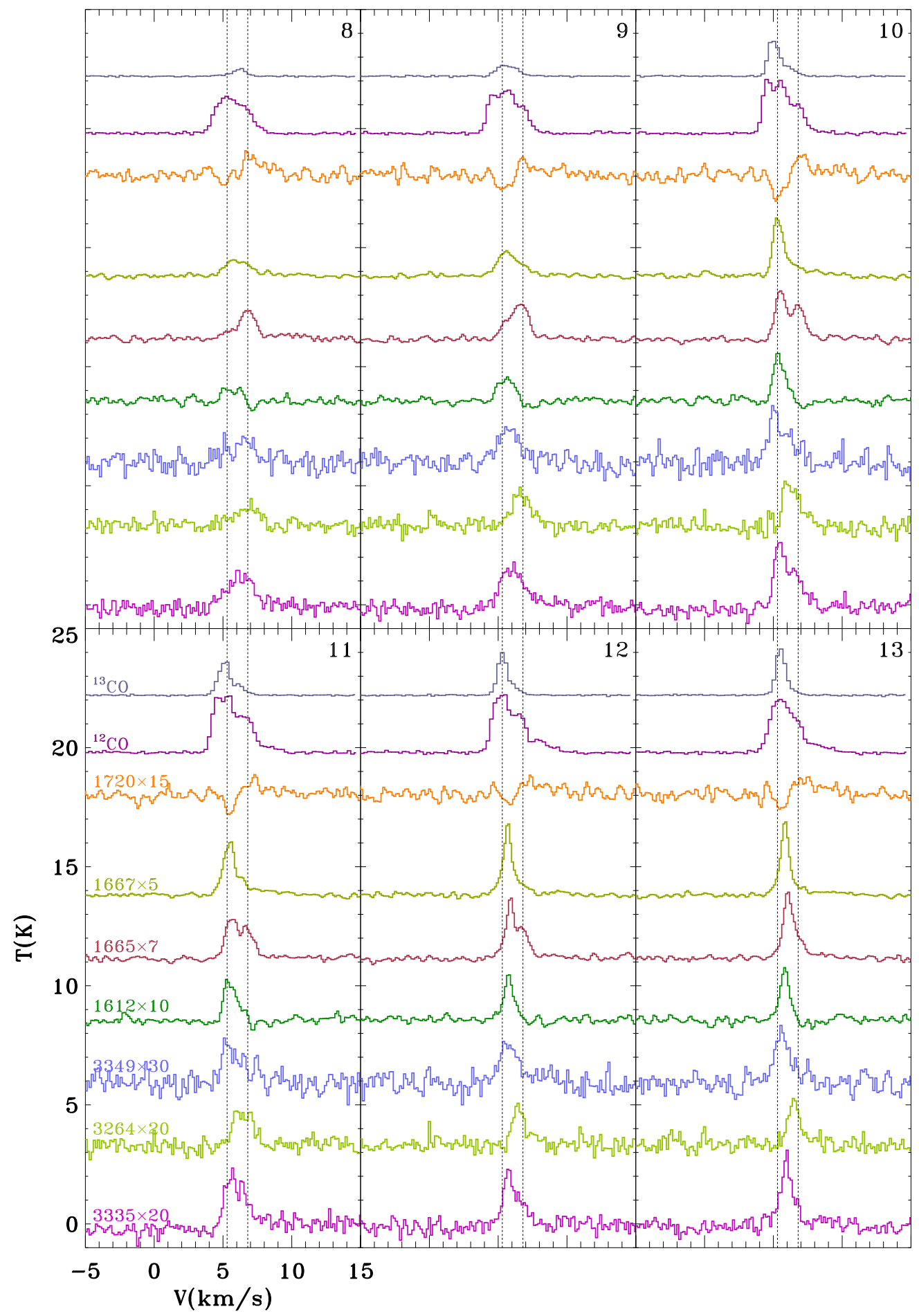

.pdf

Figure 4. Average spectra of all five cuts of $\mathrm{CH} 3335,3264,3349 \mathrm{MHz}, \mathrm{OH} 1612,1665,1667,1720 \mathrm{MHz},{ }^{12} \mathrm{CO} \mathrm{J}=1-0$, and ${ }^{13} \mathrm{CO} \mathrm{J}$ $=1-0$ at positions 8-13. The ${ }^{12} \mathrm{CO} \mathrm{J}=1-0$, and ${ }^{13} \mathrm{CO} \mathrm{J}=1-0$ spectra were convolved to the $\mathrm{OH}$ beam size of $3^{\prime}$ at each position. The vertical dashed lines indicate the central velocities of the two components of $\mathrm{OH} 1665 \mathrm{MHz}$ at position 10. 
Table 1

CH $3335 \mathrm{MHz}$ spectra parameters along the boundary

\begin{tabular}{|c|c|c|c|c|c|c|}
\hline \multirow{2}{*}{$\begin{array}{c}\text { Position } \\
\text { ID }\end{array}$} & \multicolumn{3}{|c|}{ Red Component $^{a}$} & \multicolumn{3}{|c|}{ Blue Component } \\
\hline & Height $^{b}(\mathrm{~K})$ & Center $\left(\mathrm{km} \mathrm{s}^{-1}\right)$ & Width $\left(\mathrm{km} \mathrm{s}^{-1}\right)$ & Height $^{b}(\mathrm{~K})$ & Center $\left(\mathrm{km} \mathrm{s}^{-1}\right)$ & Width $\left(\mathrm{km} \mathrm{s}^{-1}\right)$ \\
\hline 1 & - & - & - & $0.036 \pm 0.004$ & $6.5 \pm 0.1$ & $1.9 \pm 0.3$ \\
\hline 2 & - & - & - & $0.036 \pm 0.004$ & $6.5 \pm 0.1$ & $2.0 \pm 0.3$ \\
\hline 3 & - & - & - & $0.052 \pm 0.004$ & $6.6 \pm 0.06$ & $1.5 \pm 0.1$ \\
\hline 4 & - & - & - & $0.051 \pm 0.004$ & $6.7 \pm 0.06$ & $1.6 \pm 0.1$ \\
\hline 5 & - & - & - & $0.050 \pm 0.004$ & $6.5 \pm 0.07$ & $1.7 \pm 0.2$ \\
\hline 6 & - & - & - & $0.048 \pm 0.004$ & $6.5 \pm 0.07$ & $1.9 \pm 0.2$ \\
\hline 7 & - & - & - & $0.064 \pm 0.004$ & $6.3 \pm 0.06$ & $1.9 \pm 0.1$ \\
\hline 8 & - & - & - & $0.064 \pm 0.004$ & $6.4 \pm 0.06$ & $2.2 \pm 0.1$ \\
\hline 9 & - & - & - & $0.080 \pm 0.004$ & $6.0 \pm 0.04$ & $2.0 \pm 0.1$ \\
\hline 10 & $0.12 \pm 0.02$ & $5.4 \pm 0.03$ & $0.80 \pm 0.1$ & $0.075 \pm 0.005$ & $6.4 \pm 0.2$ & $1.6 \pm 0.3$ \\
\hline 11 & $0.071 \pm 0.04$ & $5.4 \pm 0.08$ & $0.90 \pm 0.3$ & $0.070 \pm 0.01$ & $6.3 \pm 0.3$ & $1.6 \pm 0.5$ \\
\hline 12 & $0.10 \pm 0.02$ & $5.7 \pm 0.05$ & $0.80 \pm 0.1$ & $0.050 \pm 0.006$ & $6.6 \pm 0.2$ & $1.3 \pm 0.4$ \\
\hline 13 & $0.13 \pm 0.005$ & $6.0 \pm 0.02$ & $1.1 \pm 0.1$ & - & - & - \\
\hline 14 & $0.12 \pm 0.005$ & $6.0 \pm 0.03$ & $1.4 \pm 0.1$ & - & - & - \\
\hline 15 & $0.098 \pm 0.004$ & $6.0 \pm 0.03$ & $1.5 \pm 0.1$ & - & - & - \\
\hline 16 & $0.098 \pm 0.005$ & $5.9 \pm 0.03$ & $1.3 \pm 0.1$ & - & - & - \\
\hline 17 & $0.11 \pm 0.005$ & $5.8 \pm 0.03$ & $1.3 \pm 0.1$ & - & - & - \\
\hline
\end{tabular}

${ }^{a}$ After Position 12, Red Component means the converged component.

$b$ The height of main beam temperature $T_{M B}$.

Table 2

$\mathrm{OH} 1665 \mathrm{MHz}$ spectra parameters along the boundary

\begin{tabular}{|c|c|c|c|c|c|c|}
\hline \multirow{2}{*}{$\begin{array}{c}\text { Position } \\
\text { ID }\end{array}$} & \multicolumn{3}{|c|}{ Red Component $^{a}$} & \multicolumn{3}{|c|}{ Blue Component } \\
\hline & Height $^{b}(\mathrm{~K})$ & Center $\left(\mathrm{km} \mathrm{s}^{-1}\right)$ & Width $\left(\mathrm{km} \mathrm{s}^{-1}\right)$ & Height $^{b}(\mathrm{~K})$ & Center $\left(\mathrm{km} \mathrm{s}^{-1}\right)$ & Width $\left(\mathrm{km} \mathrm{s}^{-1}\right)$ \\
\hline 1 & - & - & - & $0.10 \pm 0.005$ & $6.8 \pm 0.03$ & $1.2 \pm 0.1$ \\
\hline 2 & - & - & - & $0.11 \pm 0.006$ & $6.9 \pm 0.03$ & $1.1 \pm 0.1$ \\
\hline 3 & - & - & - & $0.10 \pm 0.005$ & $6.8 \pm 0.03$ & $1.4 \pm 0.1$ \\
\hline 4 & - & - & - & $0.12 \pm 0.006$ & $6.9 \pm 0.03$ & $1.1 \pm 0.1$ \\
\hline 5 & - & - & - & $0.13 \pm 0.006$ & $6.9 \pm 0.02$ & $1.0 \pm 0.1$ \\
\hline 6 & - & - & - & $0.15 \pm 0.006$ & $6.9 \pm 0.02$ & $1.2 \pm 0.1$ \\
\hline 7 & $0.06 \pm 0.01$ & $6.1 \pm 0.3$ & $1.2 \pm 0.4$ & $0.14 \pm 0.02$ & $6.9 \pm 0.07$ & $0.8 \pm 0.1$ \\
\hline 8 & $0.04 \pm 0.01$ & $5.3 \pm 0.09$ & $0.9 \pm 0.2$ & $0.17 \pm 0.01$ & $6.8 \pm 0.02$ & $1.3 \pm 0.1$ \\
\hline 9 & $0.09 \pm 0.01$ & $5.5 \pm 0.08$ & $1.0 \pm 0.1$ & $0.21 \pm 0.01$ & $6.7 \pm 0.04$ & $1.2 \pm 0.1$ \\
\hline 10 & $0.29 \pm 0.01$ & $5.5 \pm 0.01$ & $0.9 \pm 0.03$ & $0.20 \pm 0.01$ & $6.8 \pm 0.02$ & $1.1 \pm 0.1$ \\
\hline 11 & $0.23 \pm 0.01$ & $5.6 \pm 0.03$ & $1.0 \pm 0.05$ & $0.17 \pm 0.01$ & $6.8 \pm 0.04$ & $1.2 \pm 0.1$ \\
\hline 12 & $0.20 \pm 0.01$ & $5.9 \pm 0.01$ & $0.5 \pm 0.03$ & $0.19 \pm 0.01$ & $6.3 \pm 0.03$ & $1.8 \pm 0.1$ \\
\hline 13 & $0.35 \pm 0.01$ & $6.1 \pm 0.01$ & $1.1 \pm 0.02$ & - & - & - \\
\hline 14 & $0.37 \pm 0.01$ & $6.2 \pm 0.01$ & $1.0 \pm 0.02$ & - & - & - \\
\hline 15 & $0.33 \pm 0.01$ & $6.2 \pm 0.01$ & $1.0 \pm 0.02$ & - & - & - \\
\hline 16 & $0.29 \pm 0.01$ & $6.2 \pm 0.01$ & $1.0 \pm 0.02$ & - & - & - \\
\hline 17 & $0.31 \pm 0.01$ & $6.2 \pm 0.01$ & $0.9 \pm 0.02$ & - & - & - \\
\hline
\end{tabular}

${ }^{a}$ After Position 12, Red Component means the converged component.

$b$ The height of main beam temperature $T_{M B}$.

\section{CH AS A MOLECULAR GAS TRACER}

Based on dust extinction and total gas column density provided by previous studies (Pineda et al. 2010; Orr et al. 2014; Xu et al. 2016), we examine the evolution of $\mathrm{CH}$ across TBR in terms of $X$-factor. We show the correlation of the integrated intensity $W$ (in $\mathrm{K} \mathrm{km} \mathrm{s}^{-1}$ ) of $\mathrm{CH} 3335 \mathrm{MHz}, \mathrm{OH} 1665 \mathrm{MHz}$ and ${ }^{12} \mathrm{CO}$ 1-0 versus the visual extinction in Figure 8. Over a wide visual extinction range (0.4-2.7 mag), $\mathrm{W}(\mathrm{CO})$ and $\mathrm{W}(\mathrm{OH})$ correlate better with $A_{v}$ than $\mathrm{W}(\mathrm{CH})$ does. But in a more limited extinction range between 0.8 and 2.1 mag, more appropriate for a translucent cloud and/or the transition zone of a dark cloud, there is a better correlation between $\mathrm{W}(\mathrm{CH})$ and $A_{v}$ than those between $\mathrm{W}(\mathrm{OH}), \mathrm{W}(\mathrm{CO})$ and
$A_{v}$. To better examine these relationships, we adopt the usual definition of " $X$-factor",

$$
X \text {-factor }=N_{\mathrm{H} 2} / W,
$$

where $N_{\mathrm{H} 2}$ is the column density of $\mathrm{H}_{2}$ and $\mathrm{W}$ is the integrated intensity of the molecular tracers $\mathrm{CH}, \mathrm{OH}$, $\mathrm{CO}$. We plot the correlation of " $\mathrm{X}$-factor" of $\mathrm{CH}, \mathrm{OH}$ and $\mathrm{CO}$ versus the visual extinction in Figure 8. When the visual extinction $A_{v}$ is in the range between 0.8 and 2.1 mag, the $X_{\mathrm{CH}}$-factor is almost a constant of $(1.0 \pm 0.06) \times$ $10^{22} \mathrm{~cm}^{-2} \mathrm{~K}^{-1} \mathrm{~km}^{-1} \mathrm{~s}$. The dispersion of $\mathrm{CH} X$-factor is visibly less than those of $\mathrm{OH}$ and $\mathrm{CO}$ at $0.8 \leq A_{v} \leq 2.1$ mag. $\mathrm{CH}$ appears to be a better tracer of molecular gas than $\mathrm{CO}$ and $\mathrm{OH}$ in the transition zone $\left(0.8 \leq A_{v} \leq 2.1\right.$ mag). Where extinction drops below 1 mag in TBR, the 
Table 3

$\mathrm{CO}$ spectra parameters along the boundary

\begin{tabular}{|c|c|c|c|c|c|c|}
\hline \multirow{2}{*}{$\begin{array}{c}\text { Position } \\
\text { ID }\end{array}$} & \multicolumn{3}{|c|}{${ }^{12} \mathrm{CO}$} & \multicolumn{3}{|c|}{${ }^{13} \mathrm{CO}$} \\
\hline & Height $^{a}(\mathrm{~K})$ & Center $\left(\mathrm{km} \mathrm{s}^{-1}\right)$ & Width $\left(\mathrm{km} \mathrm{s}^{-1}\right)$ & Height $^{a}(\mathrm{~K})$ & Center $\left(\mathrm{km} \mathrm{s}^{-1}\right)$ & Width $\left(\mathrm{km} \mathrm{s}^{-1}\right)$ \\
\hline 1 & $1.7 \pm 0.04$ & $6.3 \pm 0.01$ & $0.9 \pm 0.03$ & $0.1 \pm 0.01$ & $6.2 \pm 0.07$ & $1.4 \pm 0.17$ \\
\hline 2 & $1.6 \pm 0.04$ & $6.4 \pm 0.01$ & $1.0 \pm 0.03$ & $0.1 \pm 0.02$ & $6.3 \pm 0.06$ & $1.0 \pm 0.14$ \\
\hline 3 & $1.4 \pm 0.04$ & $6.4 \pm 0.01$ & $1.1 \pm 0.03$ & $0.1 \pm 0.01$ & $6.4 \pm 0.07$ & $1.4 \pm 0.17$ \\
\hline 4 & $1.3 \pm 0.03$ & $6.3 \pm 0.01$ & $1.3 \pm 0.04$ & $0.2 \pm 0.01$ & $6.3 \pm 0.02$ & $0.9 \pm 0.06$ \\
\hline 5 & $1.2 \pm 0.04$ & $6.2 \pm 0.02$ & $1.5 \pm 0.06$ & $0.2 \pm 0.01$ & $6.3 \pm 0.02$ & $0.9 \pm 0.06$ \\
\hline 6 & $1.2 \pm 0.04$ & $6.1 \pm 0.03$ & $1.8 \pm 0.07$ & $0.3 \pm 0.01$ & $6.3 \pm 0.02$ & $0.9 \pm 0.05$ \\
\hline 7 & $1.6 \pm 0.03$ & $5.8 \pm 0.02$ & $2.1 \pm 0.05$ & $0.4 \pm 0.01$ & $6.2 \pm 0.02$ & $0.9 \pm 0.04$ \\
\hline 8 & $1.6 \pm 0.03$ & $5.6 \pm 0.03$ & $2.6 \pm 0.07$ & $0.3 \pm 0.01$ & $6.2 \pm 0.02$ & $1.1 \pm 0.06$ \\
\hline 9 & $1.8 \pm 0.05$ & $5.5 \pm 0.03$ & $2.5 \pm 0.08$ & $0.5 \pm 0.01$ & $5.6 \pm 0.02$ & $1.7 \pm 0.06$ \\
\hline 10 & $2.2 \pm 0.08$ & $5.4 \pm 0.04$ & $2.5 \pm 0.11$ & $1.5 \pm 0.05$ & $5.1 \pm 0.02$ & $1.1 \pm 0.04$ \\
\hline 11 & $2.3 \pm 0.08$ & $5.5 \pm 0.05$ & $2.7 \pm 0.12$ & $1.3 \pm 0.04$ & $5.1 \pm 0.02$ & $1.2 \pm 0.05$ \\
\hline 12 & $2.2 \pm 0.08$ & $5.6 \pm 0.04$ & $2.4 \pm 0.11$ & $1.7 \pm 0.04$ & $5.3 \pm 0.01$ & $0.9 \pm 0.03$ \\
\hline 13 & $2.2 \pm 0.05$ & $5.8 \pm 0.03$ & $2.2 \pm 0.07$ & $1.9 \pm 0.03$ & $5.5 \pm 0.01$ & $0.8 \pm 0.02$ \\
\hline 14 & $2.6 \pm 0.05$ & $5.7 \pm 0.02$ & $2.0 \pm 0.05$ & $2.1 \pm 0.03$ & $5.6 \pm 0.01$ & $0.9 \pm 0.01$ \\
\hline 15 & $2.6 \pm 0.06$ & $5.5 \pm 0.02$ & $2.0 \pm 0.06$ & $1.8 \pm 0.02$ & $5.6 \pm 0.00$ & $0.8 \pm 0.01$ \\
\hline 16 & $2.4 \pm 0.07$ & $5.4 \pm 0.03$ & $2.0 \pm 0.07$ & $1.4 \pm 0.03$ & $5.6 \pm 0.01$ & $0.7 \pm 0.02$ \\
\hline 17 & $2.6 \pm 0.08$ & $5.5 \pm 0.03$ & $1.8 \pm 0.07$ & $1.4 \pm 0.04$ & $5.7 \pm 0.01$ & $0.8 \pm 0.02$ \\
\hline
\end{tabular}

${ }_{a}$ The height of main beam temperature $T_{M B}$.

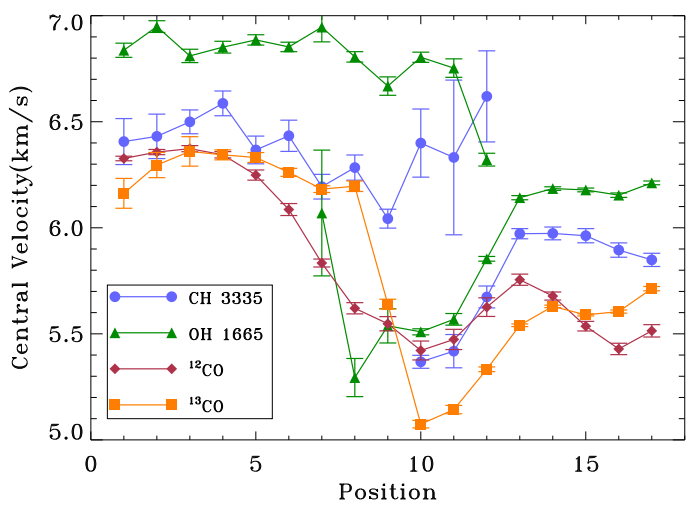

Figure 5. The change of central velocities of $\mathrm{CH} 3335 \mathrm{MHz}, \mathrm{OH}$ $1665 \mathrm{MHz},{ }^{12} \mathrm{CO} \mathrm{J}=1-0$ and ${ }^{13} \mathrm{CO} \mathrm{J}=1-0$ along the cut direction shown in Figure 2.

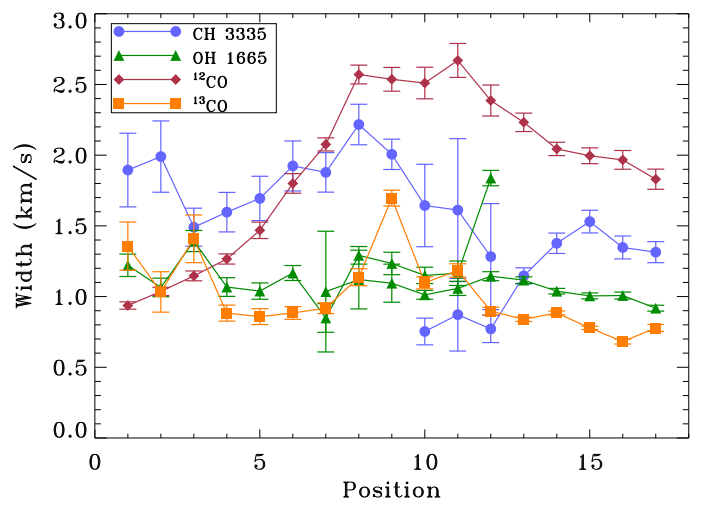

Figure 6. The change of line width of $\mathrm{CH} 3335 \mathrm{MHz}, \mathrm{OH} 1665$ $\mathrm{MHz},{ }^{12} \mathrm{CO} \mathrm{J}=1-0$ and ${ }^{13} \mathrm{CO} \mathrm{J}=1-0$ along the cut direction shown in Figure 2.

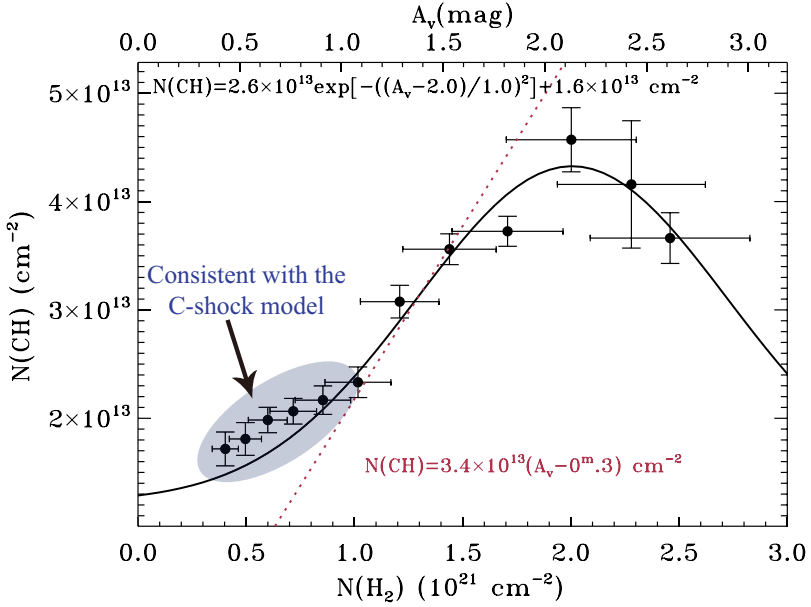

Figure 7. Change of column density of $\mathrm{CH}$ across the TBR as a function of visual extinction $A_{v}$ and total gas column density. The dashed line indicates the observational relation between the $\mathrm{CH}$ column density and visual extinction for diffuse, dark and molecular cloud (Mattila 1986). The red shade indicates the overabundance of $\mathrm{CH}$ below $1 \mathrm{mag}$ extinction. This overabundance is consistent with the C-shock model prediction.

integrated intensities $\mathrm{W}$ of the three molecules lie above the overall fitting lines, indicating a stronger intensity of these spectra, which is likely the result of C-shock in the TBR-O (Section 5).

\section{CH ABUNDANCE AND C-SHOCK MODEL}

We have calculated the column density of $\mathrm{CH}$ in Section 3.2. The column density of $\mathrm{CH}$ across the TBR is shown in Figure 7. Mattila (1986) found a good correlation between the $\mathrm{CH}$ column density and visual extinction for diffuse, dark molecular cloud $N(\mathrm{CH})=$ $3.4 \times 10^{13}\left(A_{v}-0^{m} .3\right) \mathrm{cm}^{-2}$ as the dashed line shows in Figure 7 . This correlation is reasonable considering the $\mathrm{CH}$ formation threshold, which requires substantial $\mathrm{H}_{2}$ (Black \& Dalgarno 1973). The derived column density of $\mathrm{CH}$ below 1 mag extinction (blue shade in Figure 7) in 


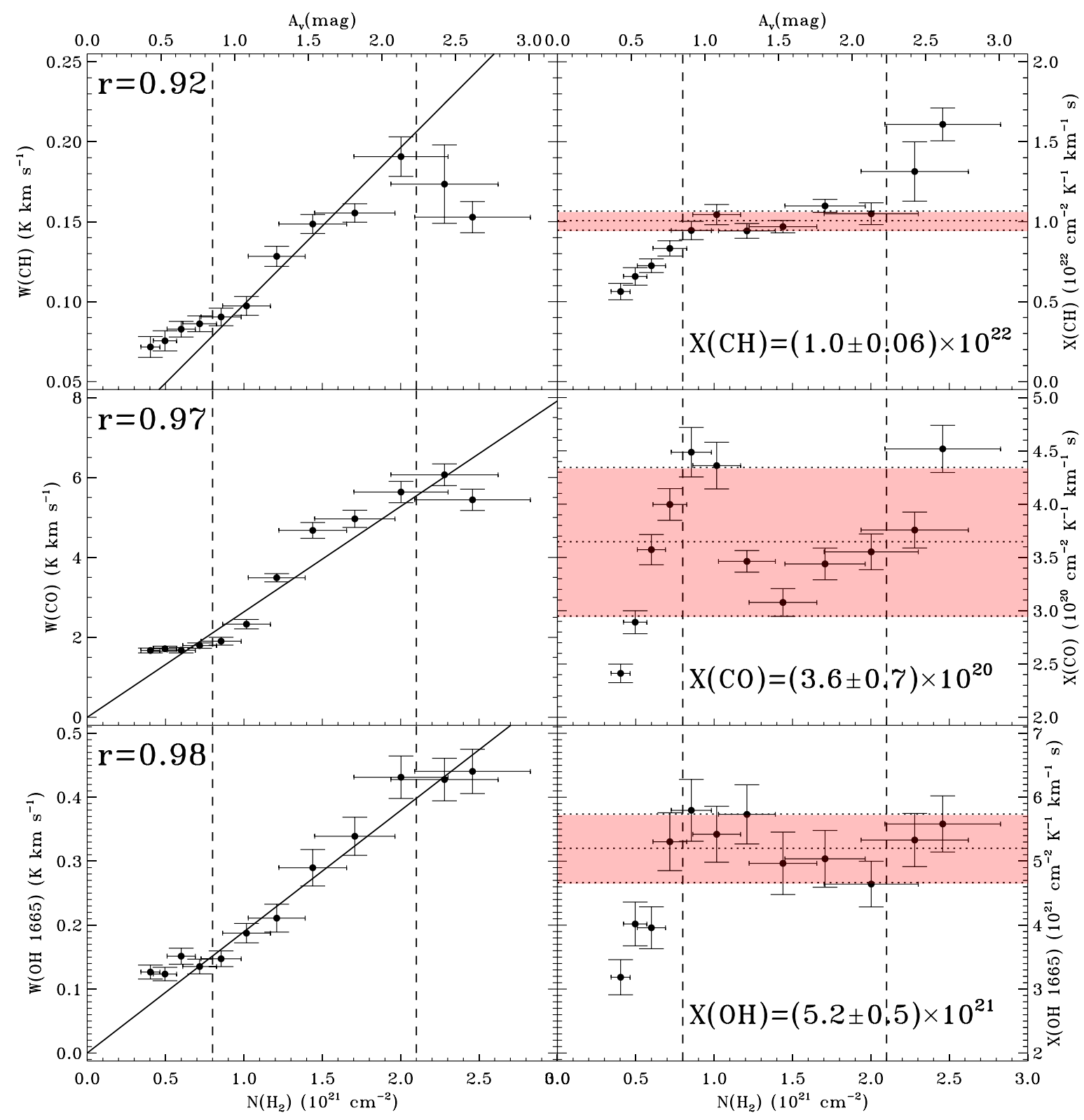

Figure 8. Correlation plot of the integrated intensity $W$ of $\mathrm{CH}, \mathrm{OH}$ and $\mathrm{CO}$ versus the visual extinction $A_{v}$, and the correlation of the "X-factor" of $\mathrm{CH}, \mathrm{OH}$ and $\mathrm{CO}$ versus the visual extinction $A_{v}$. "r" means Pearson correlation coefficient.

TBR obviously lies above the typical value observed by Mattila (1986). Furthermore, considering the correction factor for excitation temperature and optical depth (as discussed in Section 3.2), the actual column density of $\mathrm{CH}$ in TBR-O is likely to be even larger by an additional factor of 3. The true $\mathrm{CH}$ column density in TBR-O can thus be one order of magnitude higher than the simple extension from the $X$-factor for higher extinctions.

We compared the $\mathrm{CH}$ column density with the prediction of C-shock models. Flower \& Pineau des Forets (1998) made a prediction that CH column density would increase from $10^{13}$ to $10^{14} \mathrm{~cm}^{-2}$ when extinction increases from 0.1 to $1 \mathrm{mag}$ at a C-shock front, which matches exactly the measured values here. $\mathrm{Xu}$ et al. (2016) also found an overabundance of $\mathrm{OH}$ below $1 \mathrm{mag}$ extinction (by a factor of 80 ). The overabundance of $\mathrm{CH}$ and $\mathrm{OH}$ suggests that there may be an additional channel of $\mathrm{CH}$ and $\mathrm{OH}$ production, possibly due to the shock (e.g. Draine \& Katz 1986) produced by the colliding streams (Xu et al. 2016, Figure 4). When shock waves propagate through the molecular ISM, the gas is compressed, heated, and accelerated. $\mathrm{CH}$ can be produced during $\mathrm{CH}^{+}$synthesis (Equation (2)) in lower density material $\left(\sim 50 \mathrm{~cm}^{-3}\right)$ from MHD shocks (Draine \& Katz 1986; Pineau des Forets et al. 1986). In a related work, Goldsmith et al. (2010) found anomalous rotationally excited $\mathrm{H}_{2}$, indicating high gas temperature exceeding $200 \mathrm{~K}$ in TBR-O. Such high temperatures cannot be reproduced in PDR models (Goldsmith et al. 2010) while being consistent with the existence of shocks. Orr et al. (2014) also ruled out PDR models due to non-detection of [C II] in TRR-O. If temperature is above $300 \mathrm{~K}$, the neutral- 
neutral reactions become important, which can also result in the overabundance of $\mathrm{OH}$ (Neufeld et al. 2002; Xu et al. 2016).

We compared the $\mathrm{CH}$ column density with UV-driven PDR models (e.g. Levrier et al. 2012; Röllig \& Ossenkopf 2013; Morris et al. 2016). Morris et al. (2016) found that a steady-state UV-driven PDR chemistry with radiation field $\chi=(1-5) \times 10^{3}$ (in Draine units), rather than a shock chemistry, plays a key role in $\mathrm{CH}^{+}$formation in Orion BN/KL. However, the TBR has a relatively low UV field between $\chi=0.3$ and 0.8 (Flagey et al. 2009; Pineda et al. 2010). When the radiation field strength declines, the number density of $\mathrm{CH}^{+}$decreases around $A_{v}=1$ mag (Duley et al. 1992). Levrier et al. (2012) and Röllig \& Ossenkopf (2013) made a PDR model with a relatively low radiation field with $\chi=1-10$. Neither model can form as much $\mathrm{CH}$ as in our observations. In particular, Levrier et al. (2012) made predictions of $\mathrm{CH}$ column density in a UV-driven chemistry simulation with radiation field of $\chi=1$. The observed $\mathrm{CH}$ column density is still 2-3 times lager than their predictions. As discussed in Section 3.2, the actual column density of $\mathrm{CH}$ in TBR-O is likely to still be larger by another factor of 3 . The observed $\mathrm{CH}$ column density can thus be as much as 6-9 times larger than that of UV-driven simulation. UV photons should play only a limited role in producing $\mathrm{CH}$ in a relatively low radiation field such as in the TBR.

Besides the C-shock model and UV-driven PDR model, Gredel et al. (1993) and Crawford (1995) observed optical $\mathrm{CH}$ and $\mathrm{CH}^{+}$lines in several stars but found their line profiles are inconsistent with shock theories, and predict a significant velocity difference between neutral and ionized species, as discussed in Section 3.1. They believed that turbulent chemistry may play a key role in the overabundant of $\mathrm{CH}$ and $\mathrm{CH}^{+}$in these clouds. Since we do not have ionized species data (e.g. $\mathrm{CH}^{+}$), we cannot examine the proposed velocity difference between neutral and ionized species. In terms of neutral species, $\mathrm{CH}$, $\mathrm{OH}$, and ${ }^{12} \mathrm{CO}$ have mildly different central velocities (Figure 5), consistent with the propagation of C-shock. Most turbulent dissipation region models (e.g. Godard et al. 2014) predict two orders of magnitude more $\mathrm{CH}^{+}$than the PDR model, which could explain the overabundance of $\mathrm{CH}$ in TBR-O. If the region is turbulence dominated, the line width of different molecules is likely to have a linear relation. But in Figure 6, the line width of $\mathrm{CH}$, $\mathrm{OH},{ }^{12} \mathrm{CO}$ and ${ }^{13} \mathrm{CO}$ dose not show clear correlation in TBR-O, which does not support turbulence dissipation.

\section{SUMMARY AND CONCLUSIONS}

We have mapped a sharp boundary region of the Taurus molecular cloud in all three ground-state transitions of the methylidyne $(\mathrm{CH})$ radical with the Arecibo telescope. A combined analysis of $\mathrm{CH}$ data with $\mathrm{OH},{ }^{12} \mathrm{CO}$ $\mathrm{J}=1-0,{ }^{13} \mathrm{CO} \mathrm{J}=1-0$, and dust leads to the following conclusions:

1. $\mathrm{CH}$ has two kinematic components. One component shifts from 5.3 to $6 \mathrm{~km} \mathrm{~s}^{-1}$ going from outside to inside, both of which match well with those of $\mathrm{OH}$. The shifting of the two kinematic components indicates colliding streams or gas flow at the boundary region.
2. The derived $\mathrm{CH}$ abundance across the boundary is consistent with the previous observation for $0.8 \leq$ $A_{v} \leq 2.1 \mathrm{mag}$, but overabundant by as much as one order of magnitude below 1 mag extinction. The overabundance of $\mathrm{CH}$ is consistent with the prediction of the C-shock model rather than a UV-driven PDR model, which supports the existence of C-shock across the TBR. $\mathrm{CH}$ can be produced during $\mathrm{CH}^{+}$synthesis in low density material from C-shocks.

3. The scatter of $\mathrm{CH} X$-factor $(1.0 \pm 0.06) \times 10^{22}$ is much smaller than those of $\mathrm{CO}$ and $\mathrm{OH}$ in the transition zone $\left(0.8 \leq A_{v} \leq 2.1 \mathrm{mag}\right)$. $\mathrm{CH}$ is thus potentially a better tracer of molecular gas than $\mathrm{CO}$ or $\mathrm{OH}$ for translucent clouds, cloud boundaries, and the transition zone.

This work is partly supported by the China Ministry of Science and Technology under State Key Development Program for Basic Research (973 program) No. 2012CB821802, the National Natural Science Foundation of China No. 11373038, No. 11373045, and the Strategic Priority Research Program "The Emergence of Cosmological Structures" of the Chinese Academy of Sciences, Grant No. XDB09010302.

We are grateful to Paul Goldsmith for his kind and valuable advice. We would like to thank the anonymous referee for the careful inspection of the manuscript and constructive comments, particularly the important suggestion to add the comparison with the UV-driven PDR model to improve the quality of this study.

\section{REFERENCES}

Black, J. H., \& Dalgarno, A. 1973, Astrophys. Lett., 15, 79 Bujarrabal, V., Salinas, F., \& Gonzalo, I. 1984, ApJ, 285, 312 Crawford, I. A. 1995, MNRAS, 277, 458

Chastain, R. J., Cotten, D., \& Magnani, L. 2010, AJ, 139, 267

Draine, B. T., \& Katz, N. 1986, ApJ, 306, 655

Draine, B. T., \& Katz, N. 1986, ApJ, 310, 392

Duley, W. W., Hartquist, T. W., Sternberg, A., Wagenblast, R., \& Williams, D. A. 1992, MNRAS, 255, 463

Flagey, N., Noriega-Crespo, A., Boulanger, F., et al. 2009, ApJ, 701,1450

Flower, D. R., \& Pineau des Forets, G. 1998, MNRAS, 297, 1182

Genzel, R., Downes, D., Pauls, T., Wilson, T. L., \& Bieging, J. 1979, A\&A, 73, 253

Godard, B., Falgarone, E., \& Pineau des Forêts, G. 2014, A\&A, 570, A27

Goldsmith, P. F., Heyer, M., Narayanan, G., et al. 2008, ApJ, 680,428

Goldsmith, P. F., Velusamy, T., Li, D., \& Langer, W. D. 2010, ApJ, 715, 1370

Gredel, R., van Dishoeck, E. F., \& Black, J. H. 1993, A\&A, 269, 477

Hjalmarson, A., Sume, A., Elider, J., et al. 1977, ApJS, 35, 263

Indriolo, N., Blake, G. A., Goto, M., et al. 2010, ApJ, 724, 1357

Jacq, T., Baudry, A., Despois, D., Gerard, E., \& Johansson, L. E. B. 1987, A\&A, 173, 347

Lambert, D. L., \& Danks, A. C. 1986, ApJ, 303, 401

Lang, K. R., \& Wilson, R. F. 1978, ApJ, 224, 125

Levrier, F., Le Petit, F., Hennebelle, P., et al. 2012, A\&A, 544, A22

Liszt, H., \& Lucas, R. 2002, A\&A, 391, 693

Magnani, L., Lada, E. A., Sandell, G., \& Blitz, L. 1989, ApJ, 339, 244

Magnani, L., Lugo, S., \& Dame, T. M. 2005, AJ, 130, 2725

Magnani, L., \& Onello, J. S. 1993, ApJ, 408, 559

Magnani, L., Sandell, G., \& Lada, E. A. 1992, A\&AS, 93, 509

Mattila, K. 1986, A\&A, 160, 157

Morris, P. W., Gupta, H., Nagy, Z., et al. 2016, ApJ, 829, 15 
Narayanan, G., Heyer, M. H., Brunt, C., et al. 2008, ApJS, 177, 341

Neufeld, D. A., Kaufman, M. J., Goldsmith, P. F., Hollenbach, D. J., \& Plume, R. 2002, ApJ, 580, 278

Orr, M. E., Pineda, J. L., \& Goldsmith, P. F. 2014, ApJ, 795, 26

Pineau des Forets, G., Flower, D. R., Hartquist, T. W., \&

Dalgarno, A. 1986, MNRAS, 220, 801

Pineda, J. L., Goldsmith, P. F., Chapman, N., et al. 2010, ApJ, 721,686

Röllig, M., \& Ossenkopf, V. 2013, A\&A, 550, A56

Sakai, N., Maezawa, H., Sakai, T., Menten, K. M., \& Yamamoto, S. 2012, A\&A, 546, A103
Sandell, G., Magnani, L., \& Lada, E. A. 1988, ApJ, 329, 920 Sandell, G., Johansson, L. E. B., Rieu, N. Q., \& Mattila, K. 1981, A\&A, 97, 317

Sandell, G.' Hoglund, B., \& Friberg, P. 1980, A\&A, 83, 226

Sheffer, Y., Rogers, M., Federman, S. R., et al. 2008, ApJ, 687, 1075

Viala, Y. P. 1986, A\&AS, 64, 391

Xu, D., Li, D., Yue, N., \& Goldsmith, P. F. 2016, ApJ, 819, 22 